\title{
UNDERSTANDING OF THE ROLE OF pH IN FILLING MESOPOROUS SILICA WITH MAGNETIC NANOPARTICLES
}

\author{
Wiktor Wolak ${ }^{1}$, Miroslaw Roman Dudek ${ }^{1}$, Dinh Quang Ho ${ }^{1,2}$, Karol Marcjan ${ }^{1}$ \\ ${ }^{I}$ Institute of Physics, University of Zielona Gora \\ Zielona Góra, Poland \\ ${ }^{2}$ Vinh University \\ Vinh city, Nghe An, Vietnam \\ 20000621@stud.uz.zgora.pl,M.Dudek@if.uz.zgora.pl \\ hodinhquangdhv@gmail.com,karol.marcjan@gmail.com
}

\begin{abstract}
Computer simulation study of filling pores of mesoporous silica with magnetic iron oxide nanoparticles which are diffusing towards the silica surface from a water solution with a given $\mathrm{pH}$ was presented. Three different values of the $\mathrm{pH}$ of the solution were under consideration, $\mathrm{pH}=4,7$, and 10 . The size of nanoparticles was of the order of magnitude of the nanopore diameter. It was observed that in the case of a low concentration of magnetic nanoparticles in the water solution the process of filling the silica pores weakly depends on the $\mathrm{pH}$ of the solution. The value of $\mathrm{pH}$ becomes only relevant for large concentration of magnetic nanoparticles. In the simulation, a 2-state Potts model was used for preparing the silica matrix. Diffusion of magnetic nanoparticles was restricted to lattice sites only.
\end{abstract}

Keywords: mesoporous silica, magnetic nanoparticles, point of zero charge

\section{Introduction}

Recently, an increasing number of engineering applications of magnetic nanoparticles can be noted, especially those based on iron oxides which are non-toxic or where the toxicity is negligible. There are materials for medical applications like magnetic resonance [1] or drug release [2] but also new materials for industrial chemistry [3], or materials for environmental protection. In our recent papers [4, 5] we showed the special feature of iron oxide nanoparticles both experimentally and theoretically that when some amount of acid or base is added to an aqueous solution containing magnetic grains, then the solution close to the nanoparticles becomes, respectively, less acidic and less alkaline than it is expected. This property was suggested to be used in the design of new type of mortar with magnetic grains inside [5] achieving an increased compressive strength of mortar as well as its chemical protection against the acidic environment. In the following, we discuss the effect of the $\mathrm{pH}$ of water suspension of magnetic nanoparticles on filling the pores of the mesoporous silica. To this aim computer modeling methods have been used. 


\section{Preparing porous matrix}

A series of models of porous glass were introduced which were studied with the help of computer simulation methods, like the molecular dynamics method presented in papers $[6,7]$. In the following, we used the Metropolis Monte Carlo method, instead. To model porous glass, the 2-state Potts model on cubic lattice $L \times L \times L$ was chosen to simulate the phase separation of a binary mixture by analogy to a molecular dynamics approach presented in papers $[6,7]$. The following interaction hamiltonian between two kinds of model atoms A and B of the binary mixture is assumed:

$$
H=-\sum_{<i, j>} J_{1} \delta_{q_{i} q_{j}}-\sum_{<i, j>} J_{2}\left(1-\delta_{q_{i} q_{j}}\right),
$$

where lattice sites $i, j$ are occupied by atom $q$ (A or $\mathrm{B}$ ), the angular brackets denote summation over the nearest-neighbour lattice pairs. In the following, atoms A represent silica material and atoms B represent vacancies. Hamiltonian in Eq. (1) is the particular case of the hamiltonian which was introduced in paper [8] to model the sintering process in metal powders. We assume that $J_{1}>0$ and $J_{2}=0$. Initially, atoms $A$ and vacancies $B$ are randomly placed in the sites of a cubic $L \times L \times L$ lattice with a given concentration $c_{A}$ of atoms $\mathrm{A}$ and $c_{B}$ of vacancies, and $c_{A}+c_{B}=1$. During the computer simulation, atoms and vacancies which are located in the neighbouring lattice sites $i, j$ can exchange according to Metropolis rule with a success rate equal to $\min \left(1, e^{-\Delta E / k_{B} T}\right)$, where $\Delta E=E_{\text {new }}-E_{\text {old }}$ denotes the energy difference between the new and old lattice site configuration. In Figure 1, it has been shown the result of Monte Carlo simulations of a cube with dimension $L=50$ lattice sites in $\mathrm{x}, \mathrm{y}, \mathrm{z}$ direction. The periodic boundary condition was applied.

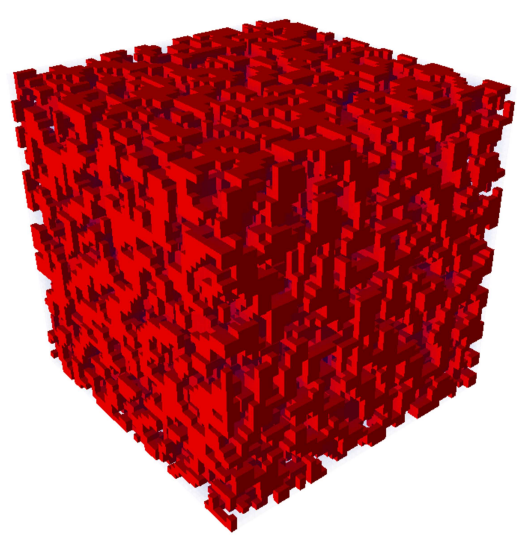

Fig. 1. Result of $N=600 \mathrm{MC}$ steps of computer simulation of binary alloy of initially randomly distributed atoms A and B on cubic $L \times L \times L$ lattice. The parameters which have been chosen: $L=50, J_{1}=0.1, J_{2}=0, T=100 \mathrm{~K}$, concentration of A atoms $c_{A}=0.56$. Atoms A (in red) represent model of porous silica 


\section{Magnetic nanoparticles suspension}

In the model, magnetic nanoparticles are represented by spheres of radius $R$ which can occupy cubic lattice sites. The cubic lattice is partitioned into two parts where the first one is represented by silica sites and the remaining sites represent a solution contacting the silica surface. Each sphere representing magnetic nanoparticle possesses magnetic dipole moment $\mu$ and a point charge $q$ in its center. The value of charge is the same for each sphere. It represents the electrostatic charge acquired by iron oxide material when it is contacting a water solution with a given $\mathrm{pH}$ value. Then, there is electrostatic repulsion between spheres as well as magnetic attraction due to the magnetic dipolar interaction. The electrostatic part of the interaction potential is the following:

$$
U_{i j}^{\mathrm{e}}=\frac{q^{2} \lambda_{D} \mathrm{e}^{-r_{i j} / \lambda_{D}}}{4 \pi \varepsilon r_{i j}},
$$

where $r_{i j}$ is the distance between the centers of mass of the nanoparticles located at sites $i$ and $j, \varepsilon$ represents permittivity, $\lambda_{D}$ is the Debye length

$$
\lambda_{D}=\sqrt{\frac{\varepsilon k_{B} T}{2 N_{A} q_{e}^{2} I}},
$$

$q_{e}$ denotes electron charge, $k_{B}$ is the Boltzmann constant, $N_{A}$ is the Avogadro number, $I$ represents ionic strength. It is assumed that the electrostatic surface potential of the nanoparticle can be approximated by Nernst potential, which depends on $\mathrm{pH}$ as follows [11]:

$$
\Psi_{s}(p H)=2.3026 \frac{R_{\mathrm{gas}} T}{F}(P Z C-p H),
$$

where $R_{\text {gas }}$ is the gas constant, $F$ is the Faraday constant and $P Z C$ represents the point of zero charge. In the following, we have chosen $P Z C=7.9$ for magnetic nanoparticles and $P Z C=2$ for porous matrix representing silica. The surface charge density is expressed as the following:

$$
\sigma=\varepsilon \frac{k_{B} T}{q_{e} \lambda_{D}}\left(2 \sinh \left(q_{e} \Psi_{s}(p H) / 2 k_{B} T\right)+\frac{4 \lambda_{D}}{R} \tanh \left(q_{e} \Psi_{s}(p H) / 4 k_{B} T\right)\right)
$$

and it is assumed that $q=4 \pi \sigma R^{2}$.

Magnetic interaction potential represents magnetic dipole-dipole interaction according to the formula:

$$
U_{i j}^{\text {dipole }}=-\frac{\mu}{4 \pi} \frac{3\left(\vec{m}_{i} \cdot \vec{r}_{i j}\right)\left(\vec{m}_{j} \cdot \vec{r}_{i j}\right)}{r_{i j}^{5}}+\frac{\mu}{4 \pi} \frac{\left(\vec{m}_{i} \cdot \vec{m}_{j}\right)}{r_{i j}^{3}},
$$


where $\vec{m}_{i}$ and $\vec{m}_{j}$ represent magnetic moments of nanoparticles at site $i$ and $j$, respectively. The interaction potentials are complemented by the Lennard-Jones interaction potential $U_{L J}$ which represents the short-ranged Van der Waals interaction, respectively the magnetic nanoparticle-magnetic nanoparticle and magnetic nanoparticle-silica in the water solution. In general, the silica surface will acquire charge $Q$ after contacting water solution with a given $\mathrm{pH}$ which is different from the value of $q$. In this case, the electrostatic interaction potential in Eq. (2) is changed to the following one:

$$
U_{i j}^{\mathrm{e}}=\frac{q Q \lambda_{D} \mathrm{e}^{-r_{i j} / \lambda_{D}}}{4 \pi \varepsilon r_{i j}} .
$$

In the model, magnetic nanoparticles in a water suspension change the orientation of the magnetic moment and jump over unoccupied cubic lattice sites according to the Metropolis algorithm where the energy term in $\Delta E$ includes all the interaction potentials. Some fraction of magnetic nanoparticles penetrates the pores of the silica surface and sticks to the surface. The concentration of nanoparticles in the solution was chosen to be equal to $0.34 \%$ to prevent agglomeration events in the solution. The diameter of pores was of the order of magnitude of the dimension of nanoparticle.
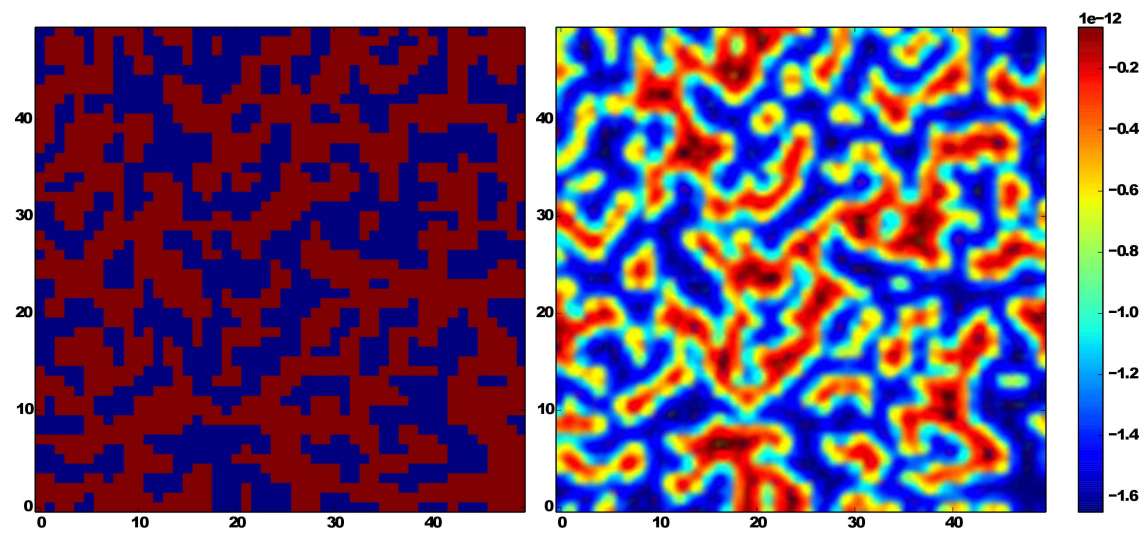

Fig. 2. On the left, a two-dimensional projection of the simulated silica surface is shown. Red and blue rectangles represent silica and pores respectively. On the right, the corresponding effective interaction potential (electrostatic and Van der Waals) is presented which is generated by the silica and felt by nanoparticle with radius $r=2.5 \mathrm{~nm}$ in a solution with $\mathrm{pH}=10$ at distance $z=5 \mathrm{~nm}$ from the silica surface

\section{Results and discussion}

Magnetic nanoparticles which are close to silica surface experience $\mathrm{pH}$-dependent electrostatic potential which strongly depends on the distance to the surface. In Figure 2, it was shown both the two-dimensional projection of the silica surface 
(left panel) and the corresponding effective interaction potential (the sum of electrostatic interaction potential and Van der Waals interaction potential) of the simulated silica at $p H=10$ at the distance $z=5 \mathrm{~nm}$ (right panel) which is experienced by a single magnetic nanoparticle with diameter of $5 \mathrm{~nm}$. The question arises to what extent the $\mathrm{pH}$ value affects the pore filling in the case when the size of the nanoparticle is comparable to the pore diameter. For such a value of $\mathrm{pH}$ as in Figure 2, magnetic nanoparticles experience strong electrostatic repulsion but still the Van der Waals attraction is a few orders higher than the electrostatic one. Our computer simulation suggests that the process of filling the silica pores by magnetic nanoparticles with dimension of the order of magnitude of the pore diameter does not depend on the $\mathrm{pH}$ value when the concentration of nanoparticles in the solution is low. Consequently, the density of nanoparticles in silica nanopores weakly depends on the value of $\mathrm{pH}$. It is evident in Figure 3, where the distribution of magnetic nanoparticles in the region close to the surface of the porous matrix has been shown for three values of $\mathrm{pH}(4,7$, and 10$)$.

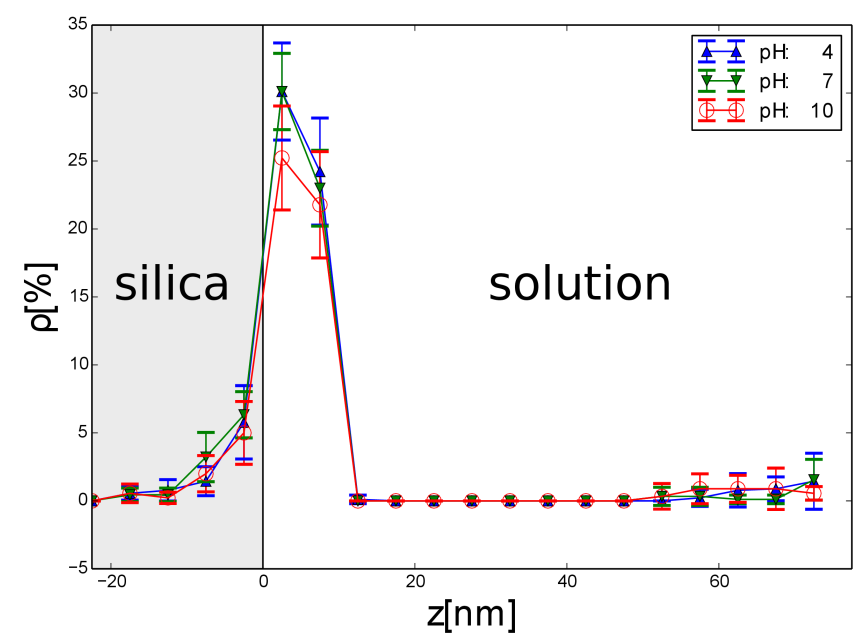

Fig. 3. Space dependence of the density $\varrho$ of magnetic nanoparticles in the region close to the surface of the porous matrix which is contacting solution with a given value of $\mathrm{pH}$ is shown. All nanoparticles have a radius equal to $r=2.5 \mathrm{~nm}$.

Three values of $\mathrm{pH}$ were chosen, $\mathrm{pH}=4,7$, and 10

There is no significant difference between the fragments of the plots corresponding to the interior of silica. The effective potential experienced by magnetic nanoparticles approaching the silica surface strongly depends on their distance to the surface. In the case of large concentration of magnetic nanoparticles, the role of the value of $\mathrm{pH}$ increases. In paper [10], the preferential filling the silica pores by the alkaline solution with magnetic nanoparticles was observed. The results strongly depend on the value of $P Z C$ of the materials under consideration. In particular, in the case of magnetic iron oxide nanoparticles can take the value between 
6.3 [5] and 7.9 [11]. In a magnetic suspension with the large concentration of magnetic nanoparticles, the dipolar magnetic interactions become important and they compete with the $\mathrm{pH}$-dependent electrostatic repulsion between the nanoparticles.

\section{Conclusions}

A computer simulation of the process of filling the nanopores of the silica glass by water magnetic suspension at given value of $\mathrm{pH}$ was under discussion. It was concluded that $\mathrm{pH}$ value is significant for the surface adsorption of the nanoparticles but filling the pores by them weakly depends on the value of $\mathrm{pH}$ in the case of low concentration of magnetic nanoparticles.

\section{Acknowledgements}

We thank Bartlomiej Zapotoczny for his discussion and comments on the work.

\section{References}

[1] Lodhia J., Mandarano G., Ferris N. J., Eu P., Cowell S.F., Development and use of iron oxide nanoparticles (Part 1): Synthesis of iron oxide nanoparticles for MRI, Biomed. Imaging and Intervention Journal 2010, 6(2), e12.

[2] Huang S., Li C., Cheng Z., Fan Y., Yang P., Zhang C., Yang K., Lin J., Magnetic $\mathrm{Fe}_{3} \mathrm{O}_{4}$ mesoporous silica composites for drug delivery and bioadsorption, J. Colloid. Interf. Sci. 2012, 376, 312-321.

[3] Lu A.H., Salabas E.L., Schuth F., Magnetic nanoparticles: Synthesis, protection, functionalization, and application, Angew. Chem. Int. Ed. 2007, 46, 1222-1244.

[4] Zapotoczny B., Dudek M.R., Koziol J.J., Mleczko J., Nanobuffering property of $\mathrm{Fe}_{3} \mathrm{O}_{4}$ magnetic nanoparticles in aqueous solution, Physica A 2013, 392, 1493-1499.

[5] Borucka-Lipska J., Kiernozycki W., Guskos N., Dudek M.R., Ho D.Q., Wolak W., Marc M., Koziol J.J., Kalaga J.K., Investigation on magnetite concentrate with respect to its use in concrete, submitted.

[6] Gelb L.D., Gubbins K.E., Characterization of porous glasses: Simulation models, adsorption isotherms, and the Brunauer-Emmett-Teller analysis method, Langmuir 1998, 14(8), 2097-2111.

[7] Gelb L.D., Gubbins K.E., Pore size distributions in porous glasses: a computer simulation study, Langmuir 1999, 15(2), 305-308.

[8] Dudek M.R., Gouyet J.F., Kolb M., Q+ 1 state Potts model of late stage sintering, Surface Science 1998, 401(2), 220-226.

[9] Keren R., Sparks D.L., Effect of $\mathrm{pH}$ and ionic strength on boron adsorption by pyrophyllite, Soil Science Society of America Journal 1994, 58(4), 1095-1100.

[10] Zapotoczny B., Dudek M.R., Guskos N., Kozio J.J., Padlyak B.V., Kosmider M., Rysiakiewicz-Pasek E., FMR study of the porous silicate glasses with $\mathrm{Fe}_{3} \mathrm{O}_{4}$ magnetic nanoparticles fillers, Journal of Nanomaterials 2012, 8.

[11] Tombacz E., Majzik A., Horvat Z.S., Illes E., Magnetite in aqueous medium: coating its surface and surface coated with it, Romanian Reports in Physics 2006, 58(3), 281-286. 